\title{
Neurotransmitter gene network reconstruction and analisis
}

Ivanov R.A.

Sector of Bioinformatics and Information Technologies in Genetics Institute of Cytology and Genetics Novosibirsk, Russian Federation ivanov.romanart@yandex.ru

Vasiliev G.V.

Sector for Genomic Studies Institute of Cytology and Genetics Novosibirsk, Russian Federation genn@bionet.nsc.ru
Matushkin Y.G.

Laboratory of Molecular-Genetic Systems

Institute of Cytology and Genetics

Novosibirsk, Russian Federation mat@bionet.nsc.ru

Savostyanov A.N

Laboratory of Psychological Genetics, Institute of Cytology and Genetics; Laboratory of Differential Psychophysiology, Institute of Physiology and Basic Medicine, Novosibirsk, Russian Federation savostyanov@bionet.nsc.ru
Klimenko A.I.

Sector of Bioinformatics and Information Technologies in Genetics

Kurchatov genomics center Institute of Cytology and Genetics Novosibirsk, Russian Federation klimenko@bionet.nsc.ru

Lashin S.A.

Sector of Bioinformatics and Information Technologies in Genetics Kurchatov genomics center Institute of Cytology and Genetics Novosibirsk, Russian Federation lashin@bionet.nsc.ru

\begin{abstract}
Research of biological bases of depression is one of the main interests of modern neurophysiology. It is still unknown how complex interaction between genes correlates to human depression behavior. Neurobiological understanding of these interactions could help to reveal the molecular mechanisms of depression. Insight on these interactions is crucial for development of diagnostic and treatment of depression. In our research, we reconstruct networks of protein-protein interaction and transcriptional regulation as well, in attempt to create the model of complex interaction between environmental and genetic risk factors of depression.
\end{abstract}

\section{Keywords - neurotransmitter, SNP, gene networks}

\section{Motivation and Aim}

The main interest of modern neuroscience is aimed at studying the biological bases of individual differences in human behavior. The medical relevance of this topic is due to the fact that development of a number of neurological and psychiatric diseases, as well as the characteristics of their occurrence, are directly related to the psychological characteristics of individuals [1]. Many papers on the mechanisms of regulation of individual behavior indicate the important role of neurotransmitter systems in the mechanisms of its regulation [2]. Also recent studies suggest that genetic factors may be modulated by environmental factors [3] which makes it important to study the environmental influences on genetic factors of human behavior. In our research we chose to focus on depression and mental diseases as the extreme manifestations of human behavior. Today gene network approaches are powerful tools for studying genes associated with human traits. In our research, we are trying to identify genes, which expression differs in different populations, based on analysis of sequencing data from several human populations.

\section{Methods}

At first, we formed the set of genes associated with neurological diseases for which the difference in several human population was shown based on an analysis of number of specialized databases, namely SFARI Gene, BDgene. PsyGeNET, epiGAD, MK4MDD and HGNC. From these set of genes, we have reconstruct networks of protein interaction using web-service StringDB. Later on we conduct the Gene Ontology analysis of our network by DAVID GO web-service [4] to select list of gene associated with neurological disease. Based on our gene network we also allocated transcriptional regulation factors using MoLoTool web-service [5] (we used significance threshold $p_{\text {ajusted }}<10-4$, which is recommended by the web-service) and reconstruct networks of transcriptional regulation. After that we build the library for targeting sequencing based on Roche's SeqCap EZ kit and conducted Illumina sequencing experiment on more than 500 people from different populations. Obtained data was analyzed via tools of bcftools to receive the list of population-related SNP for further analysis and comparison with phenotypical data.

\section{Results}

Using the methods mentioned earlier, we identified a set of genes associated with anxiety behavior and set of genes associated with mental disorders for which the difference in phenotype manifestation among different human populations was shown. Based on this sets of genes, the gene networks were reconstructed. GO analysis reveals 165 genes that were conducted in DNAseq experiment for further verification of our model. Transcriptional regulation networks revealed that our network contains 3 TF: TP53, SREBF2, SREBF1. From the DNAseq experiment on more than 500 people from various populations, we received SNP data unique for each population and soon we will conduct complex analysis of these SNP with phenotypical data of EEG and psychological questionnaires.

\section{ACKNOWLEDGMENT}

The research was partially funded by the RFBR Grant 18-29-13027 and the Budget Project 0259-2019-0008.

\section{REFERENCES}

[1] G.G. Knyazev et al. "Resting state connectivity mediates the relationship between collectivism and social cognition," Int. J. Psychophysiol., vol. 123, no. November, pp. 17-24, 2018.

[2] R.P. Ebstein, "The molecular genetic architecture of human personality: Beyond self-report questionnaires," Mol. Psychiatry, vol. 11 , no. 5 , pp. $427-445,2006$.

[3] A. N. Savostyanov et al. "Association of anxiety level with polymorphic variants of serotonin transporter gene in Russians and Tuvinians," Russ. J. Genet. Appl. Res., vol. 5, no. 6, pp. 656-665, 2015.

[4] D. W. Huang et al. "Systematic and integrative analysis of large gene lists using DAVID bioinformatics resources," no. 2, 2008.

[5] I. V. Kulakovskiy et al., "HOCOMOCO: Towards a complete collection of transcription factor binding models for human and mouse via large-scale ChIP-Seq analysis," Nucleic Acids Res., vol. 46, no. D1, pp. D252-D259, 2018. 